\title{
Pratiques
}

Linguistique, littérature, didactique

187-188 | 2020

Enseignement du texte littéraire dans l'espace

francophone: pratiques, formation, recherche

\section{Lecture littéraire et « enroulement spiralaire »: le défi de la bifurcation}

\section{Nicolas Rouvière}

\section{(2) OpenEdition \\ Journals}

Édition électronique

URL : https://journals.openedition.org/pratiques/9041

DOI : $10.4000 /$ pratiques.9041

ISSN : 2425-2042

Éditeur

Centre de recherche sur les médiations (CREM)

Référence électronique

Nicolas Rouvière, "Lecture littéraire et « enroulement spiralaire » : le défı de la bifurcation », Pratiques

[En ligne], 187-188 | 2020, mis en ligne le 12 décembre 2020, consulté le 21 juillet 2021. URL : http://

journals.openedition.org/pratiques/9041; DOI : https://doi.org/10.4000/pratiques.9041

Ce document a été généré automatiquement le 21 juillet 2021.

(c) Tous droits réservés 


\title{
Lecture littéraire et « enroulement spiralaire » : le défi de la bifurcation
}

\author{
Nicolas Rouvière
}

Dans un article intitulé "Compréhension, interprétation, explication », B. ShawkyMilcent (2020) distingue deux approches différentes de la lecture littéraire chez les didacticiens de la littérature. Si un consensus existe autour de la conception d'un va-etvient dialectique entre participation et distanciation (Dufays, Gemenne, Ledur, 2015 [1996]), ainsi que sur la nécessité d'accueillir les réactions subjectives des élèves pour permettre une appropriation des textes, la chercheuse différencie une approche «alternante » de la lecture littéraire, et une approche qu'elle qualifie d'« enroulement spiralaire ». La première, qu'elle réfère aux travaux de J.-L. Dufays (ibid.) et de S. Ahr (2013), dans le prolongement des propositions de M. Picard (1986), fait alterner « les phases tournées vers la subjectivité du lecteur et les phases réflexives », pour qu'une interprétation fondée sur l'objectivation analytique du texte dépasse la compréhension première. La seconde, représentée par les travaux de M.-J. Fourtanier, G. Langlade et C. Mazauric (2006, 2011), mais aussi de M. Goulet (2015), F. Le Goff (2013), A. PerrinDoucey (2012) et B. Shawky-Milcent (2014), considère qu'il n'y a pas de solution de continuité entre la lecture naïve et la lecture experte : «L'élève, quel qu'il soit, et dès son plus jeune âge, est apte à vivre des expériences littéraires entières, qui touchent son être dans sa globalité » (Shawky-Milcent, 2020, p. 54), parce que dans le "tissé ensemble " (Le Goff, 2013) des réactions subjectives, les interprétations objectives sont déjà là en puissance. À travers un détour par l'herméneutique de H.-G. Gadamer (1996 [1960]) et de P. Ricœur (1998 [1986]), B. Shawky-Milcent laisse entendre qu'il n'y a pas de rapport d'opposition entre ces deux approches, mais plutôt un rapport d'enveloppement de la première par la seconde. Pour H.-G. Gadamer (1996, p. 329), "l'interprétation n'est pas un acte qui s'ajoute après coup et occasionnellement à la compréhension: comprendre, c'est toujours interpréter ». Pour P. Ricœur (1998, p. 201), «la compréhension est plutôt le moment non méthodique, qui, dans les sciences de l'interprétation, se compose avec le moment méthodique de l'explication. Ce moment précède, accompagne, clôture et ainsi enveloppe l'explication. En retour l'explication développe analytiquement la compréhension ». 
2 Dans cette présentation, l'« enroulement spiralaire » comprend donc, au sens propre de " prendre ensemble », " prendre avec soi », le travail herméneutique sur la forme, mais aussi, pour B. Shawky-Milcent (2020, p.53), «la manière dont le jeune lecteur s'approprie les éclairages culturels, les pistes interprétatives, les échanges avec les pairs pour nourrir sa réception personnelle du texte, en revenant toujours au point de contact par lequel ce texte précis est entré en résonance avec son histoire et son psychisme ».

3 Je souhaiterais dans le présent article mettre au défi de la bifurcation cette conception compréhensive d'un enveloppement. Car l'enroulement spiralaire me semble pouvoir embrasser et accueillir aussi un type de dispositif qui peut présenter deux caractéristiques à priori assez éloignées des approches du sujet lecteur : d'une part la construction d'un objet de savoir à travers la découverte d'un texte documentaire contextualisant, d'autre part, un accompagnement fortement guidé de l'enseignant, pour engager les élèves à réviser l' interprétation qu'ils ont progressivement construite et consolidée au fil des séances d'étude du texte. Il s'agit en l'occurrence d'une relecture de la nouvelle «La Parure » de G. de Maupassant (1885), à la lumière d'un intertexte juridique, au cycle 4 du collège, en classe de quatrième, auprès d'élèves âgés de 13 et 14 ans.

4 Après avoir posé le cadre théorique de deux composantes de la lecture axiologique, je présenterai le dispositif et analyserai les moments de classe concernés, du point de vue des gestes de lecture et des gestes professionnels. Je discuterai ensuite la prise en compte d'un tel dispositif au titre de l'enroulement spiralaire, pour questionner en retour la didactique du sujet lecteur sur les savoirs contextuels, la démarche d'enseignement et le guidage.

\section{Formation de la pensée et génétique des valeurs : deux composantes de la lecture axiologique}

Cette réflexion s'inscrit dans la continuité des travaux en didactique qui ont contribué à une théorisation de la lecture littéraire, mais elle s'en distingue en repensant ces approches à l'aune du «tournant éthique " (Nussbaum, 2010). Dans un article intitulé «Les composantes de la lecture axiologique " (Rouvière, 2018), j'ai proposé une conception pluri-vectorielle de la lecture, en dégageant six composantes où s'actualisent les «compétences et latitudes axiologiques du lecteur» (Dufays, 1994, p. 213). Il s'agit de composantes empathique, sémiotique, appréciative, génétique et pré-philosophique, ainsi que d'une composante pré-civique qui concerne le rapport à la vérité au cours de la discussion littéraire.

6 Pour chacune de ces composantes, j'ai montré comment la réflexion sur les valeurs du texte pouvait conduire à une auto-réflexivité sur celles que l'on mobilise dans la lecture, l'articulation entre ces deux niveaux constituant une donnée transversale, intrinsèquement constitutive de la lecture axiologique. L'hypothèse est que, lorsque les scénarios pédagogiques font varier les articulations entre les différentes composantes de la lecture axiologique, on favorise d'autant mieux l'engagement des élèves dans la lecture littéraire. Il s'agit donc d'aider les enseignants à articuler dans leur pratique, avec une plus grande conscience, plusieurs de ces composantes entre elles, selon des cheminements pédagogiques diversifiés. L'article publié dans Repères se conclut par le 
postulat qu'à ces six composantes de la lecture axiologique (ouvrant la possibilité d'une réflexivité éthique sur soi) correspondent autant de champs gestuels de la lecture ${ }^{1}$.

7 Le dispositif qui est présenté ici se trouve à l'articulation de deux composantes: la composante pré-philosophique de formation de la pensée et la composante sur la génétique des valeurs.

\section{La composante pré-philosophique de formation de la pensée}

8 La perspective axiologique invite à considérer la lecture littéraire comme une activité en partie (pré)philosophique, car le lecteur devient en quelque sorte le théâtre d'un débat sur les normes et les valeurs. Ce dernier s'opère dans un constant dédoublement subjectif et mobilise les trois compétences du "philosopher » définies par M. Tozzi (2011) : la conceptualisation, la problématisation et l'argumentation.

\section{La conceptualisation}

9 Conceptualiser, c'est « rassembler sous un même sujet logique tous les éléments qui ont une même propriété générale essentielle » (Leleux \& Rocourt, 2010, p. 79). Un concept peut être défini en compréhension et en extension. Par exemple, pour définir en compréhension la notion de valeur, N. Heinich (2017) distingue la valeur comme grandeur, la valeur comme «bien» (ou objet de considération), et la valeur comme principe de valorisation. En extension, elle dénombre 16 "registres» de valeur. J.C. Guerrini (2015, p. 258) définit les valeurs-principes, qu'il appelle les " maître-mots ", comme les «garants au nom desquels on s'estime habilité à porter des jugements d'approbation ou de blâme, à justifier une préférence, à prendre position, à agir ». La question « au nom de quoi... ? » peut jouer le rôle d'extracteur, pour s'assurer que l'on a bien affaire à un concept permettant de justifier une préférence, une prise de position ou une action. J.-C. Guerrini répertorie 231 mots-valeurs. Il distingue de surcroit cinq strates de «relief axiologique » dans le discours, de l'axiologie implicite à l'axiologie explicite. Car les mots-valeurs «se trouvent constamment à l'horizon de l'activité axiologique, y compris dans des discours qui prétendent en être complètement émancipés » (ibid., p. 284).

10 La capacité à nommer ces maitres-mots est étroitement liée à celle de nommer les émotions, qui sont des "perceptions de valeur» (Tappolet, 2000), en ce qu'elles reposent sur un système intériorisé de buts, de normes et de préférences (Charaudeau, 2000). Dans «La Parure», Mathilde Loisel est traversée par différents sentiments, comme l'orgueil, la honte ou le sentiment d'humiliation. Le sentiment intériorisé de la honte la conduit au début du récit à prendre ses distances avec son amie riche, Mme Forestier, puis la perspective d'être bien habillée pour le bal la rehausse suffisamment dans sa propre estime, pour qu'elle ose aller la solliciter. Ainsi peut-on inférer de son comportement différentes valeurs, comme la dignité, l'estime de soi, mais aussi plus tard une forme d'honnêteté et de loyauté, dans la résolution de payer sa dette jusqu'au bout.

11 Les ressources du lecteur pour la conceptualisation sont ses connaissances langagières, ses connaissances expérientielles et ses connaissances culturelles. Mais le lecteur trouve aussi des aides à l'intérieur de la fiction elle-même : tout d'abord, face à des mots inconnus, il est souvent possible, grâce au contexte, de trouver des substituts 
approchants. Ensuite, par des procédures de redondance, bien analysées par S. Suleiman (1983) dans le roman à thèse, la fiction met souvent en scène plusieurs champs d'application ou plusieurs scènes d'actualisation d'une même notion, ce qui peut aider à sa conceptualisation.

Par ailleurs c'est dans les contrastes, les oppositions thématiques, les effets de surprise ou les conflits que se perçoit le mieux la «différence axiologique». Ceci est particulièrement marqué dans le genre de la nouvelle durant la période 1870-1925. F. Goyet (1993) a montré comment ces récits étaient polarisés par une double structure antithétique, la seconde, plus latente, n'étant révélée qu'au moment de la chute. Dans "La Parure ", l'opposition entre la dignité sociale et la honte, qui tourmente la protagoniste, est supplantée à la fin par l'opposition entre l'illusion et la réalité, lorsque l'on découvre que le collier est un faux. Cela invite à relire la nouvelle en prêtant attention aux indices qui pointent les fausses représentations de Mathilde, son idéalisation de la haute société, sa logique d'essentialisation qui lui fait confondre les beautés de l'apparat et le vrai.

\section{La problématisation des normes et des valeurs}

13 Si l'on considère que le propre de la littérature est de jouer à suspendre les catégories préexistantes, à les brouiller ou les reconfigurer, la seconde grande opération de pensée est la problématisation.

Au niveau du monde fictionnel, il est possible de dégager quelques questions morales et civiques essentielles: 1) Au nom de quelles valeurs individuelles et collectives les personnages agissent-ils? 2) Quelles conséquences peuvent avoir leurs choix d'action? 3) Quelles sont les responsabilités individuelles et collectives ? 4) Quels facteurs sociaux ont pu conduire à de telles situations? Par exemple dans "La Parure ", quelles conséquences peut avoir le choix de mentir à Mme Forestier pour gagner du temps? Au nom de quoi Mme Loisel prend-elle ensuite le parti de travailler durement pour rembourser la dette du couple? Le schéma péché-rédemption, dévoyé dans un sens matérialiste, travaille discrètement le texte à travers plusieurs connotations religieuses ironiques (rue des "Martyrs", "se perdre ou se sauver", se contempler dans "sa gloire», "Champs Elysées»). Qui est responsable de l'endettement du couple? Mme Loisel parce qu'elle a perdu le collier, ou le mari parce qu'il fait le choix de cacher la vérité ? Enfin quels facteurs sociaux peuvent expliquer l'aveuglement des époux sur la nature du bijou ? Au-delà du bovarysme de la protagoniste, lié à son statut de femme mal mariée, on découvre à posteriori combien le poids des hiérarchies sociales engendre un cloisonnement des représentations. Le mode de vie de la classe supérieure ne peut pas être réellement connu, il ne peut être que fantasmé, essentialisé, comme s'il conférait un supplément d'être, de telle sorte que la possibilité du faux n'est même pas envisageable.

Les mêmes questions peuvent se poser sur le plan de l'autorité énonciative. Au nom de quoi, à l'ouverture de la nouvelle, est-il affirmé ceci :

[...] car les femmes n'ont point de caste ni de race, leur beauté, leur grâce et leur charme leur servant de naissance et de famille. Leur finesse native, leur instinct d'élégance, leur souplesse d'esprit sont leur seule hiérarchie, et font des filles du peuple les égales des plus grandes dames. (Maupassant, 1885) 

cet énoncé selon les trois sphères épistémologiques de la vérité de J. Habermas (2001) ainsi que le préconise C. Leleux (2014), cela conduit à se poser les questions suivantes : 1) Cet énoncé prétend-il à l'exactitude ? Autrement dit, peut-on affirmer qu'«il est exact que la beauté naturelle soit le seul critère de hiérarchie effectif entre les femmes »? 2) Cet énoncé prétend-il à la justesse normative ? Autrement dit peut-on affirmer que «la beauté naturelle devrait être le seul critère de hiérarchie entre les femmes »? 3) Cet énoncé prétend-il à l'authenticité ? Autrement dit peut-on affirmer qu'« il est souhaitable et préférable que la beauté naturelle soit le seul critère de hiérarchie entre les femmes »?

17 À travers une formulation à valeur de vérité générale tout à fait ambigüe, le narrateur fait passer un jugement évaluatif personnel pour une norme qui devrait être. Comme le lecteur fait confiance à priori au narrateur comme dépositaire d'une autorité fiable, cela le prédispose à comprendre les frustrations de Mme Loisel. Cette dernière, parce qu'elle est belle, pense qu'elle aurait dû faire un mariage plus avantageux pour elle socialement. Et le narrateur semble penser également que cela serait préférable et plus juste pour une jolie femme. Mais le narrateur est-il fiable ? Est-ce aussi le point de vue de Maupassant, ou une stratégie narrative de sa part? conséquences sociales cela a-t-il d'écrire ceci? Enfin quels facteurs historiques et sociaux ont pu conduire à produire un tel énoncé ? Transposées au niveau du lecteur, ces questions engagent une auto-réflexivité morale et civique. Au nom de quoi agissons-nous? Quelles conséquences peuvent avoir nos choix d'action? Quels facteurs sociaux peuvent influencer nos choix dans la vie?

Ces questions nous conduisent ainsi vers une autre composante de la lecture axiologique, qui est la génétique des valeurs.

\section{La génétique des valeurs : d'où viennent les valeurs en jeu dans le texte et sa lecture?}

20 La dimension génétique intervient lorsqu'on se demande d'où viennent les valeurs (morales, sociales, politiques, religieuses ou philosophiques), que l'on identifie à la lecture du texte. Cette question rejoint les débats sur l'intentio auctoris, l'intentio operis et l'intentio lectoris, dont U. Eco (1992, p. 29-32) a clairement exposé les termes.

21 Pour établir les codes et horizons historico-culturels où s'originent les valeurs perçues au cours de la lecture, on peut s'inspirer de la typologie proposée par J.-L. Dufays (1994, p. 106) pour les stéréotypes. Les valeurs sont ainsi référables : 1) à l'auteur (biographie, statut, personnalité) ; 2) au contexte historique et social au moment de la production ; 3) à des valeurs trans-historiques à caractère anthropologique ; 4) à la fortune critique émanant des lectures successives de l'œuvre ; 5) Au contexte de réception (état de la culture) ; 6) à la personne du lecteur (biographie, sensibilité, personnalité, bibliothèque intérieure).

Ainsi le propos liminaire tenu sur les femmes pourrait-il tout d'abord être imputé aux conceptions personnelles de Maupassant, homme à femmes s'il en est, chez qui la galanterie le dispute à l'obsession sexuelle, tout comme la misogynie le dispute à l'adoration du beau sexe (Martinez, 2012)2. 
Mais il est aussi possible d'imputer les valeurs de ce propos à la doxa conservatrice des lecteurs du journal mondain Le Gaulois, le journal de l'aristocratie et de la haute bourgeoisie, dans lequel la nouvelle est publiée le 17 février 1884. Les lecteurs masculins avaient toutes les raisons de légitimer une telle vision de la femme pour accueillir, au nombre des maitresses potentielles, de jolies femmes mariées qui aspirent à s'élever socialement. Du reste pourquoi un prince charmant serait-il regardant sur les différences sociales? En même temps le propos est suffisamment ambigu pour être reçu comme une flatterie ou un hommage, par les femmes du monde qui lisent aussi le journal. Lecteurs et lectrices pouvaient en tout cas se réjouir de voir mis en scène dans la fiction, un cloisonnement social qui les protège dans la réalité, et d'en découvrir un tel degré d'intériorisation chez des gens modestes à l'horizon limité.

Reste que le propos tenu sur les femmes renvoie aussi à une certaine réalité juridique. Dans le code civil de 1804, si les femmes ne sont pas à proprement parler assimilées à des mineurs, comme le sont les "interdits ${ }^{3}$ ", en revanche une série importante de restrictions est appliquée aux femmes mariées, aux femmes célibataires et aux filles mères, quant à l'exercice de leurs responsabilités de personnes majeures ${ }^{4}$. L'épouse n'est pas une personne juridique autonome. Elle est placée sous l'entière dépendance de son mari à qui elle doit obéissance (art. 213). Rappelons quelques interdictions qui sont faites aux femmes mariées: interdiction d'accès aux lycées et aux universités; interdiction de signer un contrat et de gérer ses biens; interdiction de travailler sans l'autorisation du mari ; interdiction de toucher soi-même son salaire ; contrôle du mari sur la correspondance et les relations; interdiction de voyager à l'étranger sans autorisation; absence d'autorité parentale sur ses enfants (c'est le père qui est seul responsable de leur éducation); répression très dure de l'adultère pour les femmes (emprisonnement de 3 mois à 2 ans). Le mariage est soumis au consentement du père, sans limite d'âge. De son côté, la femme non mariée, même majeure, ne peut faire partie du conseil de famille ni exercer de tutelle sur des membres en difficulté. Elle ne peut pas non plus être témoin dans les actes d'État-civil ni dans les actes privés. Quant aux filles-mères et aux enfants naturels, la recherche de paternité étant interdite (art. 340), ils n'ont aucun droit et ne bénéficient d'aucune aide publique. À quoi s'ajoute, pour les femmes, l'exclusion totale des droits politiques.

Avec un tel statut juridique, on pourrait comprendre pourquoi le narrateur considère que «les femmes n'ont point de caste ni de race » : à titre compensatoire, il ne leur reste en effet que leur beauté native pour seule distinction. Relu au regard de ces éléments, le texte comporterait ainsi une dimension un peu plus compréhensive qu'on ne l'aurait cru, en dépit de son caractère satirique à l'égard de la protagoniste et de son couple. Il s'inscrit en tout cas dans la réflexion de l'époque sur les droits de la femme, comme le droit des filles à l'instruction publique (Loi Grévy-Ferry, 1879) et le droit au divorce (Loi Naquet, 1884).

\section{Le cadre de l'expérimentation}

Dans le cadre de la recherche "Littérature et valeurs ${ }^{5}$ ", l'expérimentation menée vise à étudier l'émergence de "gestes de lecture axiologique ", grâce à des dispositifs spécifiques, mis en place en collaboration avec les enseignants. Elle constitue un ballon d'essai en vue d'une recherche-action de plus grande envergure. Il s'agit d'une expérimentation sur le terrain avec et par les praticiens, qui vise à générer des 
connaissances, contribuer au développement des participants et induire du changement dans les pratiques (Guay, Prud'homme \& Dolbec, 2016). "capricieuse », " exigeante», " elle se plaint tout le temps», " elle veut trop la facilité », " elle se compare tout le temps à sa copine ", "c'est une obsession ", " elle est jalouse », " elle fait pas d'effort », " elle en demande trop », " elle en veut toujours plus », "elle a honte de son mari ${ }^{10} »$, etc. Ce jugement négatif a été conforté par la comparaison avec le conte de Cendrillon, motivée par le départ précipité du bal. Au terme de la déchéance sociale du couple, la chute a été masquée, dans le but de ménager l'effet de surprise, mais aussi pour faire réfléchir les élèves sur les réflexions rêveuses de Mathilde Loisel, qui pourraient constituer à ce stade une morale provisoire : «Comme la vie est singulière, changeante, comme il faut peu de chose pour vous perdre ou vous sauver ». Les élèves ont reformulé cette idée dans un sens fataliste (« la vie est trompeuse », « la vie est fragile et il faut un rien pour la changer en bien ou en mal »), mais aussi dans le sens d'une morale du renoncement (« il faut se contenter de ce qu'on a au lieu d'en demander encore plus »). Ce dernier point a été discuté («Alors est-ce que c'est mal de vouloir plus?»), certains ont dit que « quand on est très pauvre faut un peu vouloir plus sinon on gâche sa vie », et la classe est arrivée à l'idée qu' « il ne faut pas que ce soit une obsession », qu'il faut « un juste milieu ».

La surprise de la chute a fait fortement réagir les élèves : c'est " choquant », " triste ", "désespérant», "frustrant», «ça fout la haine ». Mais la morale qu'ils tirent de l'histoire, pour autant ne change pas : «Fallait pas se plaindre », « ne pas mentir », « Il faut se satisfaire de ce qu'on a », " la richesse ça en valait pas la peine », " Dire la vérité ça fait peur mais faut le faire ». Et si Mme Loisel « a trop l'seum », « fait pitié » ou « fait de la peine », elle est punie pour avoir été « égoïste », " égocentrique », " capricieuse », etc.

Le dispositif mis en place à l'avant dernière séance consiste alors à poser la question des responsabilités individuelles et collectives : comment les Loisel ont-ils pu être aussi aveugles sur la situation ? Comment en sont-ils arrivés là ? « À qui la faute? ». L'objectif est d'examiner les responsabilités possibles des autres personnages comme celles du mari ou de Mme Forestier, mais surtout de se demander si des facteurs sociaux pourraient expliquer le comportement du couple. Les élèves ont proposé des pistes de responsabilités, puis ils ont été répartis par groupe de 4 ou 5 élèves afin d'examiner dans le texte chacune des hypothèses avancées : 1) la faute de Mathilde ; 2) la faute du mari ; 3) la faute de Mme Forestier ou du Ministre ; 4) la faute des inégalités et des écarts de richesse («les personnes riches» selon la formule d'un élève). Enfin le professeur a donné à deux groupes d'élèves un intertexte juridique : une liste des restrictions du droit des femmes mariées, dans le code Napoléon de 1804. L'échange où 
la classe propose des pistes de responsabilité est retranscrit en Annexe 1. Le lancement de la consigne de recherche figure en Annexe 2 et celle spécifique aux deux groupes qui travaillent sur le code civil en Annexe 3. La fiche documentaire sur les droits de la femme mariée distribuée aux élèves figure en Annexe 4.

C'est le professeur qui a décidé de la composition des groupes. Si ces derniers sont volontairement hétérogènes, il a tout de même effectué une différenciation. Ainsi le groupe qui travaillait sur le personnage de Mathilde était plutôt composé d'élèves moyens ou en difficulté. L'un des deux groupes travaillant sur le texte documentaire était plutôt composé d'élèves moyens, l'autre composé d'élèves moyens ou forts. Ce sont les échanges au sein de ce second groupe que nous allons examiner, le premier ayant eu plus de difficultés à entrer dans le texte documentaire, pour des raisons lexicales mais aussi éthiques que nous évoquerons.

\section{L'analyse des gestes de lecture et des gestes professionnels}

L'analyse qui suit porte sur les échanges qui ont eu lieu au sein du second groupe travaillant sur le statut de la femme mariée ${ }^{11}$. Il est composé de trois garçons et une fille.

\begin{tabular}{|l|l|}
\hline 18 & P. Alors vous, vous travaillez sur quelle hypothèse ? \\
\hline 19 & E.1. La société, le code Napoléon. \\
\hline 20 & P. D'accord, ça vous surprend, de lire ça, de voir ce que la femme n'avait pas le droit de faire? \\
\hline 21 & E.1. Oui, ben oui. \\
\hline 22 & P. Et du coup Mme Loisel, comment vous pensez qu'elle se comporte et qu'elle agit ? \\
\hline 23 & E.2 ...Rébellion \\
\hline 24 & E.3 On comprend qu'elle ait voulu être riche pour avoir un minimum de droits. \\
\hline 25 & E.4 Pour avoir de l'argent. \\
\hline 26 & $\begin{array}{l}\text { P. Est-ce qu'elle a le droit de s'acheter toute seule quelque chose, de s'acheter... un manteau par } \\
\text { exemple? }\end{array}$ \\
\hline 27 & E.3 Elle peut pas. \\
\hline 28 & $\begin{array}{l}\text { E.4 C'est pas que l'argent qu'elle peut pas... c'est, c'est même que si elle a de l'argent, il faut } \\
\text { quand même l'autorisation du mari. }\end{array}$ \\
\hline 29 & P. Hé oui, même si elle a de l'argent il faut l'autorisation du mari. \\
\hline 30 & E.1 Mais peut-être que comme ça elle est heureuse? \\
\hline 31 & E.2 Oui, mais elle peut pas se faire plaisir. \\
\hline 32 & E.1 Ben si, peut-être que son mari il est gentil, et si, puisqu'on nous dit qu'elle a eu sa robe. \\
\hline 33 & E.4 Il lui a donné les 400 euros mais... \\
\hline 34 & $\begin{array}{l}\text { E.3 Mais bon on peut quand même dire que le mari, il avait heu.... il avait... il avait la main sur } \\
\text { la femme. }\end{array}$ \\
\hline [les élèves se reportent au document sur le code Napoléon] \\
\hline
\end{tabular}




\begin{tabular}{|c|l|}
\hline 35 & E.2 Elle peut rien faire, mais elle doit quand même payer des impôts. \\
\hline 36 & E.3 Elle doit signer un contrat, pour gérer ses biens. \\
\hline 37 & E.1 Comparé à la situation du mari... Elle a pas le droit... \\
\hline 38 & E.2 Comme les criminels et les débiles mentaux. \\
\hline 39 & E.4 C'était vraiment des... les femmes c'était vraiment des... \\
\hline 40 & E.1 Si elle a un enfant, l'enfant elle peut pas s'en occuper vu qu'il appartient au mari. \\
\hline 41 & E.3 Bon ben, les femmes c'était les plus faibles. Elles étaient soumises à la société. \\
\hline
\end{tabular}

33 Durant ce premier moment de verbalisation sur le texte documentaire, un élève confirme que ses camarades et lui ont été surpris (21), un autre (re)qualifie le comportement de Mathilde, en proposant le concept de rébellion (23). Les élèves disent comprendre ses motivations et se mettent donc à la place du personnage (24-25). Ils décrivent sa situation (27-28), puis ils débattent sur la façon dont Mme Loisel vit sa condition (30-34). Ils tirent d'abord des arguments de la nouvelle elle-même (30 à 33), avant que l'un ne tranche le débat en se référant au document contextuel (34). À partir de là tous se réfèrent au document qu'ils ont sous les yeux. Ils reformulent à leur propre initiative les droits et les interdictions de la femme mariée (35 à 41), montrant une compréhension explicite du document.

Ce premier moment montre des gestes qui articulent plusieurs composantes de la lecture axiologique : engagement empathique (se mettre à la place du personnage pour pouvoir ensuite le juger), formation de la pensée (conceptualisation, argumentation), génétique des valeurs (découverte et appropriation du contexte normatif). La découverte de ce document constitue sans doute un petit événement de lecture. Les élèves expriment une réaction émotionnelle (21), ils discutent d'un problème (sans indépendance, une épouse peut-elle être heureuse, même si son mari est bon avec elle ?), mais surtout à partir du tour 35 , ils font retour par eux-mêmes vers le texte documentaire et s'approprient les informations, sans que le professeur donne l'impulsion. Ce dernier intervient du reste assez peu. Il pose une question fermée sur la réaction émotionnelle des élèves, qui précadre la réponse (20), il leur demande de juger l'action du personnage de Mathilde (22), puis il pose une question sur le document (26) et valide un premier élément de compréhension (29).

Le moment d'échange suivant concerne le retour au texte de Maupassant. L'objectif est de faire le lien avec le discours liminaire du narrateur sur les femmes, afin que les élèves repèrent la présence d'un système de valeurs dominant et comprennent l'attitude de Mme Loisel.

\begin{tabular}{|l|l|}
\hline 42 & P. Est-ce qu'il y a un passage dans le texte où on parle des femmes, en général ? \\
\hline 43 & E.3 Là ? \\
\hline 44 & $\begin{array}{l}\text { P. Non dans le texte de Maupassant : regardez au début de la nouvelle, au tout début au tout } \\
\text { début. }\end{array}$ \\
\hline 45 & E.3 Elle avait une vie motonone... \\
\hline 46 & E.2 « Pas de dot, pas d'espérance... » \\
\hline 47 & P. Regardez le deuxième paragraphe, le narrateur il dit quelque chose sur les femmes. \\
\hline
\end{tabular}


E.3 «Elle fut simple ne pouvant être parée, mais malheureusement comme une déclassée, car les femmes...»

49 P. Ah! vas-y lis, « car les femmes... »

E.3 « car les femmes n'ont point de caste ni de race, leur beauté, leur grâce et leur charme leur servant de naissance et de famille. Leur finesse native, leur instinct d'élégance, leur souplesse d'esprit, sont leur seule hiérarchie, et font des filles du peuple les égales des plus grandes dames ».

51 E.2 Waouh

52 E.4 En gros ça veut dire, elles s'appartiennent même pas.

53 E.3 Elles ont pas de classe sociale, mais en gros c'est en fonction de leur beauté quoi.

54 P. Voilà, c'est ça.

55 E.3 C'est peut-être pour ça qu'elle veut des sous pour s'acheter une robe.

56 E.4 C'est compréhensible.

P. Le narrateur, ben effectivement il est en train de dire que la seule chose qui classe les femmes c'est la beauté, c'est pas l'appartenance sociale.

58 E.1 Si elle est riche et si elle est belle, en fait c'est moins...

P. Qu'est-ce que vous en pensez de cette idée que la seule façon de classer les êtres c'est leur beauté ? quand il s'agit des femmes...

60 E.3 Je trouve ça rétrograde.

61 P. Oui, pourquoi?

62 E.3 Ben parce que tu peux être pas beau et plus intelligent que quelqu'un qui est beau.

63 E.4 Ouais franchement.

64 E.2 Puis des fois les riches ils sont plus bêtes.

P. Tout à fait ... et au début le narrateur il dit que la seule chose qui compte, pour les femmes, c'est la beauté... Est-ce que ça vous semble heu... normal, de dire ça?

66 E1, E2, E3, E4 : Non/Non non non/pas du tout.

67 P. Et Mme Loisel, elle est plutôt jolie ou plutôt...?

68 E.1 Ben plutôt jolie.

P. Voilà, donc si elle est jolie, et si la société pense la même chose que ce qui est écrit ici, est-ce qu'elle a des raisons de vouloir être plus... plus regardée, plus...

70 E.4 Ben oui.

71 P.... plus admirée, plus riche...

72 E.4 Ben oui.

73 P. Du coup est-ce qu'elle a tort de vouloir... paraître?

74 E.2 Ben non, non.

P. Ça peut se comprendre, ça peut un peu se comprendre, si on considère les choses comme ça, que... seule la beauté permet de ranger les gens en haut ou en bas.

76

E.1 Elle, elle a vraiment toutes ces choses à respecter, si elle avait été riche, et ben au moins, au moins.. 


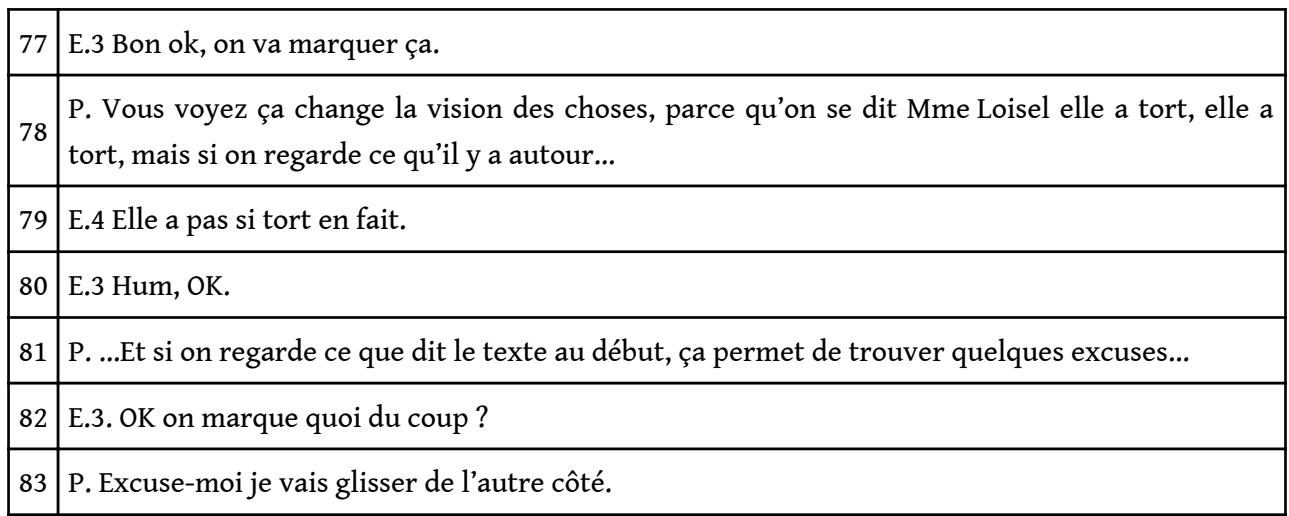

C'est le professeur qui impulse à trois reprises ce mouvement de retour au texte de Maupassant $(42,44,47)$, jusqu'à pointer le passage précis et le faire lire à voix haute (49). Ce passage n'a pas fait l'objet auparavant d'un questionnement dans la séquence, mais comme il se situe au tout début du texte, l'élève qui commence à relire les premières phrases tombe rapidement sur ces lignes. Un élève réagit émotionnellement (51), deux autres reformulent spontanément la conception qui est donnée du statut de la femme $(52,53)$, ce qui les conduit ensemble à reconsidérer les « raisons » de Mathilde Loisel $(55,56)$. Le professeur valide à deux reprises la compréhension par ces élèves du discours du narrateur $(54,57)$. Dans la suite de l'échange, il s'applique du reste à en reformuler l'idée principale, afin que le propos de fond soit bien compris $(57,59,65$, 75). Au tour 59, il demande aux élèves ce qu'ils pensent de ce discours général; il acquiesce à une première réponse (65) et relance encore sa demande en demandant aux élèves de porter un jugement sur le propos du narrateur (65). Le professeur recentre ensuite le questionnement sur le personnage de Mathilde en demandant aux élèves de juger son action (67). C'est clairement lui qui guide le questionnement logique. Il laisse une première fois transparaître son opinion qui est en accord avec celle des élèves (65), puis fait avancer la réflexion par des questions fermées $(65,67,69,73)$, jusqu'à synthétiser de façon nuancée leur avis commun selon lequel Mathilde a des circonstances atténuantes (75). Durant cette phase, les gestes de lecture des élèves n'en sont pas moins riches: ils portent un jugement empathique sur les motivations de Mathilde (59), puis portent un jugement négatif sur le discours général du narrateur $(60,66)$, contre lequel ils argumentent $(62,63,64)$, avant de reconsidérer les actions du personnage (70-72-74). L'impact émotionnel de cette découverte résonne encore dans le propos de l'un d'eux (76), et débouche sur une modification explicite du jugement porté sur Mme Loisel $(79,80)$. Le professeur tire une conclusion métacognitive, sur le fait qu'on a changé de vision sur le personnage (78), grâce à la connaissance du contexte (78) et grâce à la relecture du texte littéraire (81), permettant ainsi une réflexivité des élèves sur l'acte de lecture.

\section{Discussion}

Un tel dispositif peut-il être compté au titre de «l'enroulement spiralaire » appelé de leurs vœux par les didacticiens sensibles à la cause du sujet-lecteur? Certains ne manqueront pas de répondre par la négative. Car il est clair que le professeur a d'emblée en tête une lecture du texte qui est la sienne et qu'il entend transmettre aux élèves: Mathilde Loisel a des circonstances atténuantes, en raison du 
statut de la femme à l'époque et en raison du système général de valeurs dont le texte porte la trace. Et c'est ce contexte qui peut en partie expliquer les frustrations, les illusions et les humeurs de la protagoniste. Voilà le projet de lecture et la signification à transmettre. L'ensemble du dispositif est construit pour parvenir logiquement à cette conclusion et faire adhérer les élèves à la lecture du professeur. On serait donc dans un cas d'instrumentalisation du texte littéraire, voire de manipulation de la lecture des élèves, aux antipodes d'une lecture qui partirait de leur subjectivité et s'enrichirait par différents gestes appropriatifs entrant en résonance avec l'histoire et le psychisme des sujets. À ce compte, peu importe l'habileté mise en œuvre pour masquer la chose et faire comme si l'interprétation partait des élèves. Tout est faussé dès le départ, et l'on pourrait même s'inquiéter des dérives possibles de cette démarche, qui viserait moins à accompagner le processus de lecture qu'à faire adhérer les élèves à un discours préexistant, comme par exemple à l'interprétation selon laquelle l'aveuglement des personnages serait imputable au cloisonnement hiérarchique des groupes sociaux. À l'appui de cette critique, on peut citer le refus d'un élève d'adhérer au pré-cadrage du sens opéré par le professeur, au moment de la consigne de recherche. Cet élève, qui faisait partie du premier groupe devant travailler sur le statut de la femme, a refusé le principe même d'une responsabilité possible de la société. Nous retranscrivons en annexe 5 ce moment d'échange, qui a mobilisé dans le groupe concerné toute la rhétorique persuasive du professeur, sans que pour autant l'élève change d'avis ${ }^{12}$.

Il me semble cependant que ce type de dispositif mériterait d'être pris en considération au titre de l'enroulement spiralaire, dans la continuité des théories du sujet lecteur. Tout d'abord sa mise en place intervient en toute fin de séquence et ne prend sens qu'au regard de l'ensemble des séances qui ont précédé. Or l'étude a largement favorisé jusque-là les gestes appropriatifs, dans une triple direction psychoaffective, éthique et sociologique. Le choix du dévoilement progressif a permis aux élèves d'imaginer les réactions de Mathilde lorsqu'elle reçoit l'invitation au bal, de porter ensuite un jugement sur sa réaction inattendue. Les élèves ont pu dire s'ils partageaient le sentiment de cette dernière, selon lequel « il n'y a rien de plus humiliant que d'avoir l'air pauvre au milieu des femmes riches ». Un travail lexical a permis de distinguer les notions de fierté, d'orgueil, de vanité, de honte et d'humiliation. Les élèves ont également été sollicités pour inventer une suite narrative après la phrase «Le Ministre la remarqua ", qui marque l'acmé du succès de Mathilde lors du bal. Il s'agissait d'une stratégie de la fausse piste, pour mieux cerner par la suite combien la réalité sociale, déceptive et cruelle, était aux antipodes des scénarios d'ascension qu'on aurait pu imaginer. Les élèves ont présenté leurs différents scénarios, qui allaient du divorce avec le mari, au mariage avec le Ministre, en passant par le crime conjugal. La trouvaille du lien intertextuel avec le conte de Cendrillon a permis par contraste de mieux ancrer le récit dans la veine réaliste. Ils ont discuté du choix de mentir sur la perte du bijou, et complété le tableau sociologique des différents groupes sociaux qui avait été entamé à la séance deux. Jusqu'à la découverte de la chute, il s'avère que les élèves sont allés dans le sens de ce que le texte programme largement, tout en mobilisant leurs propres valeurs : ils ont critiqué Mme Loisel sur un plan psychologique et moral, ils ont parfois éprouvé de la pitié pour ce qu'elle était devenue. Même après la découverte de la chute, une morale du renoncement a continué à se faire entendre, sur le tort d'avoir voulu s'élever et paraitre. Autrement dit le dispositif ici présenté n'a pris place que lorsque le sens, pour les élèves, semblait entendu et bouclé. Il s'agissait alors de renverser la perspective, pour modifier le regard des élèves sur la protagoniste, et montrer par là- 
même que les significations d'un texte littéraire excèdent toujours la lecture première qu'on peut en faire. Cette stratégie du contrepied est du reste à l'image du mécanisme de la chute, qui conduit à relire le texte différemment. Ce retournement constitue un certain forçage par rapport à la lecture première et il est donc compréhensible que la démarche soit guidée. Le but n'est pas de transmettre une interprétation qui est celle du lecteur expert, il est de relancer la lecture sur de nouvelles bases. Chaque groupe a mené son enquête pour juger des responsabilités des uns et des autres, en exhumant de nouvelles traces, ce qui a donné lieu à une synthèse des rapporteurs à l'oral. D'ailleurs, le dispositif aurait pu permettre en fin de séquence de déboucher sur un sujet argumentatif, du type «Pensez-vous que Mme Loisel soit responsable de son propre déclassement?». Tous les arguments discutés collectivement à l'oral auraient pu en effet être affichés et classés, de telle sorte que le travail écrit aurait surtout consisté en une mise en forme structurée de la réponse. Peu importe alors la nature des responsabilités individuelles ou sociales que les élèves auraient bien voulu retenir. On ne les juge pas sur leur opinion, mais sur l'étayage de leur argumentation.

Le second argument qui légitime, selon moi, le fait de ne pas négliger ce dispositif au titre de l'enroulement spiralaire, c'est que le détour intertextuel par le document juridique a permis un véritable retour au texte et le questionnement d'un passage que les élèves n'avaient pas remarqué de prime abord. A contrario, à quoi servirait la contextualisation, si elle n'aidait pas à problématiser la lecture ? Dans la perspective de B. Shawky-Milcent (2020, p. 53), il s'agit bien de «s'approprier les éclairages culturels, les pistes interprétatives, les échanges avec les pairs pour nourrir sa réception personnelle du texte ». Comment le détour par un document contextuel ne serait-il pas du reste un peu plus guidé ? Les élèves ne peuvent pas inventer un tel support, d'autant que la pertinence de son lien avec le texte est tributaire de la seule lecture experte du professeur. Par ailleurs on est bien revenu à la question des aspirations et des frustrations sociales de Mme Loisel, le «point de contact » par lequel ce texte est entré en résonance avec la sensibilité des élèves. Cette question s'est enrichie d'une lecture contextuelle, sociologique et historique.

41 En retour, il me semble que ce dispositif pourrait interroger la didactique du sujet lecteur sur plusieurs points. Le premier est la façon d'envisager le contexte sociohistorique qui entoure la production des œuvres. La didactique du sujet lecteur a déplacé le centre de gravité de l'enseignement, en s'attachant moins aux objets de savoir textuels et contextuels qu'à la réception et l'appropriation des textes. Elle s'est donc intéressée aux théories de la lecture actualisante, plus dynamisantes, à la fois pour questionner les textes à partir des préoccupations actuelles et pour créer un vaet-vient différentiel et critique avec le contexte historique. Dans le dispositif présenté ici, la stratégie de contextualiser après-coup s'avère payante en elle-même pour problématiser la lecture, sans besoin de recourir à des questions actualisantes, précisément parce qu'à la première lecture, on ne pense pas spontanément à l'écart historique qui existe avec le statut de la femme aujourd'hui.

42 Le second point est qu'une stratégie de manipulation de la lecture par le professeur a trouvé sa légitimité. Ici cette stratégie a relancé la lecture interprétative du texte et a permis d'exhumer de nouvelles traces. Le dernier point a trait à la question de la directivité dans l'enseignement du professeur. Y. Daumet (2018) se demande si une certaine doxa de la non-directivité ne tendrait pas à se constituer en sur-moi pédagogique. La volonté très forte de respecter l'interprétation des élèves pourrait 
conduire certains professeurs à éviter de proposer une direction d'interprétation que les élèves n'auraient pas formulée eux-mêmes. Il émet l'hypothèse selon laquelle ce principe pédagogique s'imposerait aux enseignants avec encore plus de rigueur dans le domaine éthique, en raison d'une confusion avec le principe de neutralité axiologique.

À l'appui de cette hypothèse, je souhaiterais évoquer la réponse d'un professeur, dans le cadre d'un questionnaire d'enquête qui a été soumis en 2019 aux professeurs de français de collège et lycée des académies de Lyon, Grenoble et Créteil ${ }^{13}$. À partir d'un texte donné, ces derniers devaient formuler des objectifs d'apprentissage pour la classe et une problématique de lecture de leur choix. La réponse qui m'a interpelé est la suivante :

Je ne propose pas de problématiques: je pars de leurs impressions, je demande simplement ce qu'ils ont compris du texte et à partir de là on construit ensemble une problématique qui pourrait être : « Quel message critique ce texte contient-il et comment est-il rendu efficace?»

On ne peut que se réjouir d'une démarche collaborative de construction d'un questionnement sur le texte à partir des premiers éléments de compréhension réunis avec les élèves. Cependant je m'interroge sur le fait que le professeur, dans sa réponse, s'abstienne d'indiquer dans le sondage quelle est sa propre lecture problématisée. Et je me demande si ce retrait, au nom du respect de la parole des élèves et des interprétations individuelles, ne risquerait pas de dériver vers un retrait des objectifs de lecture du professeur lui-même. Or il me semble que les enseignants peuvent s'autoriser à mettre en œuvre des dispositifs qui sont seuls tributaires de leur lecture experte, comme celui que nous venons de présenter.

Cette préoccupation s'inscrit dans le cadre d'une conception de la lecture littéraire pensée dans la continuité des théories du sujet lecteur et gagnée à la conception de l'enroulement spiralaire pour que les textes s'inscrivent durablement dans la bibliothèque intérieure et la mémoire individuelle. Mais elle procède d'un déplacement de perspective lié au questionnement axiologique. L'objectif de cette approche n'est pas de transmettre un système de valeurs ni de modifier l'échelle de valeurs des élèves, il est d'ouvrir, dans leur esprit, grâce à la fiction, le spectre des échelles de valeurs possibles, d'essayer d'en comprendre les origines potentielles et de discuter avec eux des normes d'action qui peuvent en découler. Nous faisons l'hypothèse que cette approche peut conduire à une auto-réflexivité qui sera d'autant plus subjectivante, pour la formation de la personne, si le scénario pédagogique parvient à articuler les composantes empathique, sémiotique, appréciative, génétique, et (pré)philosophique de la lecture, ainsi que la dimension (pré)civique de la discussion littéraire. Cela suppose des objectifs d'apprentissage ainsi qu'une stratégie pédagogique et didactique, dans la façon de présenter et de questionner les textes. Certains dispositifs peuvent ainsi mettre l'enroulement spiralaire au défi de la bifurcation, en particulier en termes de détour et de guidage. C'est sans doute l'occasion de requestionner les gestes professionnels, à la croisée du pédagogique et du didactique, et de considérer les représentations d'arrière-plan qui les sous-tendent, pour trouver le juste équilibre entre retrait, accompagnement, guidage et engagement. 


\section{BIBLIOGRAPHIE}

AHR, S. (2013). Vers un enseignement de la lecture littéraire au lycée. Expérimentations et réflexions. Grenoble : Scéren-CRDP.

BUCHETON, D. (1999). « Les postures du lecteur ». In : Demougin P., Massol J-F.(dirs). Lecture privée et lecture scolaire. Grenoble : CRDP, p. 201-214.

BUCHETON, D. (2006). « Les postures d'écriture et de lecture : la diversité des modes de penserparler-apprendre ». Langages \& pratiques 37, p. 29-39. En ligne : http://www.ac-grenoble.fr/ ien.vienne2/IMG/pdf_revue_arld.pdf.

Charaudeau, P. (2000). « Une problématisation discursive de l'émotion ». In : Plantin, C., Doury, M. \& Traverso, V. (dirs). Les Émotions dans les interactions. Lyon/Bron : Presses universitaires de Lyon/ Application des recherches sur la communication et les interactions, p. 125-155.

Collin, M. (2015). « Les Femmes dominées dans le code Napoléon ». La Revue nouvelle 5. En ligne : https://www.revuenouvelle.be/Les-femmes-dominees-dans-le-code-Napoleon.

DAHHAN, P. (1996). Guy de Maupassant et les femmes. Luneray : Berthout.

DAUMET, Y. (2018). «Éthique et littérature dans le corpus TALC : une histoire empêchée ?». Séminaire Littéval, Littérature et valeurs en classe : quels gestes de lecture, quels gestes professionnels?. INSPE de Grenoble, 21 nov. 2018. En ligne : http://ouvroir-litt-arts.univ-grenoble-alpes.fr/ programmes/littvaleurs/seance-1-du-seminaire-liteval

DUFAYS, J-L. (1994). Stéréotype et lecture. Essai sur la réception littéraire. Bruxelles : P. Lang.

DUFAYS, J-L., GEMENNE, L. \& LEDUR, D. (2015) [1996]. Pour une lecture littéraire. Histoire, théories, pistes pour la classe. Louvain-la-Neuve : De Boeck.

ECO, U. (1992) [1990]. Les Limites de l'interprétation. Trad. de l'italien par M. Bouzaher. Paris : Grasset.

FOURTANIER, M-J., LANGLADE, G. \& MAZAURIC, C., (2006). « Dispositifs de lecture et formation des lecteurs $» .7^{e}$ Rencontres des chercheurs en didactique de la littérature. IUFM de Montpellier, 6-8 avr. 2006. En ligne : http://perso.ens-lyon.fr/jean-charles.chabanne/didlit/

FourtanierLangladeMazauric.pdf.

FOURTANIER, M-J., LANGLADE, G. \& MAZAURIC, C., (dirs) (2011). Le Texte du lecteur. Bruxelles : P. Lang. GADAMER, H-G. (1996) [1960]. Vérité et méthode, les grandes lignes d'une herméneutique philosophique. Trad. de l'allemand par E. Sacre et al. Paris : Éd. du Seuil.

GOULET, M. (2015). « Les lieux de la lecture : du braconnage au bricolage ». In : Dezutter, O., Falardeau, E. (dirs). Les Temps et les lieux de la lecture. Namur : Presses de l'université de Namur, p. 271-305.

GOYET, F. (1993). La Nouvelle, 1870-1925. Paris : Presses universitaires de France.

GUAY, M.-H., PRUDHOMME, L., \& DOLBEC, A. (2016). « La recherche-action ». In : Gauthier, B. \& Bourgeois, I. (dirs). Recherche sociale. De la problématique à la collecte de données. Québec: Presses universitaires du Québec.

GUERRINI, J.-C. (2015). Les Valeurs dans l'argumentation. Structures axiologiques et dimensions axiologiques des disputes. Thèse de doctorat en sciences du langage : Université Lumières-Lyon 2. 
HABERMAS, J. (2001) [1999]. Vérité et justification. Trad. de l'allemand R. Rochlitz. Paris : Gallimard.

HEINICH, N. (2017). Des valeurs. Une approche sociologique. Paris : Gallimard.

LARRIVÉ, V. (2014). Du bon usage du bovarysme dans la classe de français. Développer l'empathie fictionnelle des élèves pour les aider à lire les textes littéraires : l'exemple du journal de personnage. Thèse de doctorat en lettres : Université Michel de Montaigne-Bordeaux III. En ligne : https:// tel.archives-ouvertes.fr/tel-01155167.

LELEUX, C. (2014). « Instruire et éduquer sur fond d'éthique ». Pratiques 163-164. En ligne : https:// journals.openedition.org/pratiques/2237.

LELEUX, C. \& Rocourt, C. (2010). Pour une didactique de la morale et de la citoyenneté. Développer le sens moral et l'esprit critique des adolescents. Louvain-la-Neuve : De Boeck.

LE GOFF, F. (2013). « Fiction énonciative et imaginaire du lecteur dans la formation d'une bibliothèque patrimoniale ». In : Denizot, N. \& Ahr, S. (éds). Les Patrimoines littéraires à l'école, usages et enjeux. Namur : Presses universitaires de Namur, p. 99-118.

MARTINEZ, F. (2012). Maupassant. Paris : Gallimard.

MAUPASSANT, G. (de) (1885). « La parure ». In : Contes du jour et de la nuit. Paris : Marpon/ Flammarion, p. 73-96. En ligne : https://gallica.bnf.fr/ark:/12148/bpt6k1031357/f77.item. nussbaum, M. (2010). La Connaissance de l'amour. Essais sur la philosophie et la littérature. Trad. de l'anglais par S. Chavel. Paris : Les éditions du Cerf.

OLLIER, C. (1975). « Geste de lecture ». Pratiques 7-8, p. 21-23. En ligne : https://www.persee.fr/doc/ prati_0338-2389_1975_num_7_1_920.

PERRIN-DOUCEY, A. (2012). « Apprentissage de la lecture et construction précoce d'une identité de lecteur ». Thèse de doctorat en didactique de la littérature : Université de Grenoble. En ligne : https://hal.archives-ouvertes.fr/tel-01083079/document.

PICARD, M. (1986). La Lecture comme jeu. Essai sur la littérature. Paris : Les éditions de Minuit. RICœUR, P. (1998) [1986]. Du Texte à l'action. Essais d'herméneutique II. Paris : Éditions du Seuil. ROUVIÈRE, N. (2018). « Les composantes de la lecture axiologique ». Repères 58, p. 31-47. En ligne : https://journals.openedition.org/reperes/1692.

SHAWKY-MILCENT, B. (2014). L'Appropriation des cuvres littéraires en classe de Seconde. Thèse en littératures française et francophone : Université Grenoble-Alpes. En ligne : https://tel.archivesouvertes.fr/tel-01677062.

SHAWKY-MILCENT, B. (2020). « Compréhension, interprétation, explication ». In : Brillant-Rannou, N. et al. (dirs). Un Dictionnaire de didactique de la littérature. Paris : H. Champion, p. 51-55.

SULEIMAN, S. R. (1983). Le Roman à thèse ou l'autorité fictive. Paris : Presses universitaires de France. TAPPOLET, C. (2000). Émotions et valeurs. Paris : Presses universitaires de France. TODOROV, T. (1984). Critique de la critique. Un roman d'apprentissage. Paris : Éditions du Seuil. Tozzi, M. (2011) [1994]. Penser par soi-même. Initiation à la philosophie. Lyon : Éd. Chronique sociale.

\section{ANNEXES}




\section{Annexe $n^{\circ} 1$}

Recueil des propositions des élèves qui énoncent des pistes de responsabilités

\begin{tabular}{|l|l|}
\hline 1 & $\begin{array}{l}\text { P. Alors, à qui la faute ? Proposez des idées. Comment ça se fait qu'ils en arrivent là ? On } \\
\text { propose des idées sans aller jusqu'à argumenter. Ce qui m'intéresse c'est de proposer des pistes } \\
\text { et puis après, chaque groupe s'emparera d'une piste. }\end{array}$ \\
\hline 2 & E. C'est la faute de Mathilde. \\
\hline 3 & $\begin{array}{l}\text { P. Alors, c'est la faute de Mathilde. Je pense que là-dessus, tout le monde a des idées. Est-ce qu'il } \\
\text { y aurait, peut-être, une autre part de responsabilité } ?\end{array}$ \\
\hline 4 & E. La faute au mari parce que c'est lui qui... \\
\hline 5 & $\begin{array}{l}\text { P. Alors, la faute au mari. Certains pensent que ça pourrait être la faute au mari - on va laisser } \\
\text { certains choisir. Est-ce qu'il y aurait d'autres pistes? }\end{array}$ \\
\hline 6 & E. Bah, l'amie. \\
\hline 7 & P. La faute à l'amie, pourquoi pas, on explorera. D'autres idées? \\
\hline 8 & E. La faute au ministre? \\
\hline 9 & E. Mais nan, c'est la faute à Mathilde ! \\
\hline 10 & $\begin{array}{l}\text { P. Attendez, attendez ! Carla, l'idée c'est pas de débattre pour l'instant sur ce qu'on est } \\
\text { persuadé, c'est de lancer des pistes. La faute au ministre, pourquoi pas, on pourra essayer } \\
\text { d'explorer cette piste. Oui ? }\end{array}$ \\
\hline 11 & $\begin{array}{l}\text { E. La faute à Mathilde. } \\
\text { marche. Alors, donc on va faire un travail de groupe. }\end{array}$ \\
\hline 12 & $\begin{array}{l}\text { P. Ça on l'a déjà dit. Pour l'instant je lance toutes les pistes. Donc on m'a dit - Mathilde, le mari, } \\
\text { l'amie, le ministre,... Est ce qu'il y a pas autre chose qui pourrait faire que le collier a disparu ? }\end{array}$ \\
\hline 13 & E. La malchance \\
\hline 15 & E. La malchance, oui, donc le hasard, la fatalité. Oui ? \\
\hline
\end{tabular}

\section{Annexe $\mathrm{n}^{\circ} 2$}

\section{Formulation de la consigne pour chercher dans le texte les éléments qui étayent l'hypothèse sur les responsabilités des uns et des autres.}

Alors, tout le monde écoute! L'idée, c'est de chercher dans le texte... Tout le monde écoute ? L'idée est de chercher dans le texte, «La Parure », tous les éléments qui nous montrent que l'hypothèse qu'on a formulée peut se vérifier.

Donc tout dans le texte, sur Mathilde, qui me montre qu'effectivement, la réaction ou l'attitude de Mathilde, c'est... c'est la faute de Mathilde. Tous les éléments, toutes les 
attitudes, tout ce qu'on nous dit sur le mari pour essayer de voir si ça peut être la faute du mari. Tous les éléments sur, donc, vous m'avez dit les riches - c'est à dire le fait qu'on ait une société séparée entre les différentes classes sociales. Donc tout ce qu'on nous dit sur la différence entre les différentes classes sociales qui nous montrent que, peut-être, la société a ses torts. On explore aussi la piste de - est-ce que ça peut être la faute du ministre ? Est-ce que peut être la faute de l'amie?

\section{Annexe $\mathrm{n}^{\circ} 3$}

\section{Formulation de la consigne pour découvrir le statut de la femme à l'époque de Mme Loisel et mieux comprendre sa situation.}

Et vous avez... On a vu l'idée de la société, vous avez parlé des riches. J'ai donné un document à certains groupes qui nous montrent quel statut a la femme, comment est considérée la femme à cette époque. Qu'est-ce que c'est qu'être femme à cette époque. Parce que vous connaissez certains éléments, vous vous doutez peut-être de certains éléments. Vous en avez peut-être vu certains en histoire. Donc j'ai donné un document qui leur permet de dire, ben voilà, la femme au XIXe siècle, à cette époque, voilà ce qu'elle avait comme statut, voilà ce qu'elle pouvait faire, voilà ce qu'elle ne pouvait pas faire. Et ça nous explique, peut-être aussi, pourquoi est-ce que Mathilde réagit comme ça, pense comme ça, et pourquoi est-ce que l'auteur imagine son personnage comme ça. D'accord. On me reformule ce qu'on doit faire? Quel travail vous devez faire?

\section{Annexe $n^{\circ} 4$}

\section{Contenu du document distribué aux élèves}

\section{Le code Napoléon}

Rédigé en 1804, le Code Civil des Français, dit «Code Napoléon », affirme l'incapacité juridique totale de la femme mariée :

- interdiction d'accès aux lycées et aux universités ;

- interdiction de signer un contrat, de gérer ses biens ;

- exclusion totale des droits politiques;

- interdiction de travailler sans l'autorisation du mari ;

- interdiction de toucher elle-même son salaire ;

- contrôle du mari sur la correspondance et les relations ;

- interdiction de voyager à l'étranger sans autorisation ;

- répression très dure de l'adultère pour les femmes (emprisonnement de 3 mois à 2 ans)

- les filles-mères et les enfants naturels n'ont aucun droit.

- le mariage est soumis au consentement du père

Le code civil français de 1804 limite les droits juridiques de la femme mariée, ce qui la rapproche du statut de mineur, comme les criminels et les «interdits » (personnes dérangées mentalement). 


\section{Citation de Napoléon :}

«L'enfant appartient au mari de la femme comme la pomme au propriétaire du pommier. [...] La femme est donnée à l'homme pour qu'elle lui fasse des enfants ; elle est sa propriété comme l'arbre à fruits est celle du jardinier " (Napoléon, d'après Emmanuel de Las Cases, Mémorial de Sainte-Hélène, 1822-1823)

\section{Annexe $n^{\circ} 5$}

\section{Échanges liminaires dans le second groupe qui travaille sur les droits de la femme mariée}

$\mathrm{P}:$ Attendez, attendez. Est-ce que vous pouvez répéter ce que vous venez de dire là ?

E1 : Elle a rien fait la société. Enfin je sais pas, c'est pas la faute de la société si Mathilde elle est arrogante.

E2 : Oui, mais c'est la faute de la société si...

E1 : Si elle a son caractère là ?

E1 : Je vois pas comment la société pourrait influencer Mathilde.

E4 : Bah si, puisqu'elle veut toujours être riche et que la richesse...

E1 : Bah on nous dit qu'elle sort pas, donc...

E2 : Bah si parce que la société... Genre par exemple Mathilde, elle est quand même pas pauvre mais elle est pas riche. Donc il y a des gens riches... Ah, j'arrive pas à expliquer ce que je veux dire, ça m'énerve. Faut que je trouve comment dire.

P : L'idée c'est que la société a fait en sorte qu'il y ait des pauvres et des riches, c'est ça ?

E2 : J'arrive pas à dire ce que je voulais dire...

$\mathrm{P}$ : Mais c'est intéressant. C'est très intéressant votre débat là. Parce que vous vous dites, elle sort pas, donc la société n'a pas d'influence.

E2 : Bah oui, c'est ça. Enfin...

$\mathrm{P}$ : Est-ce que vous n'êtes pas influencés, vous, par la société?

[Les élèves acquiescent]

$\mathrm{P}$ : Montrez-moi un exemple.

E4 : Les modes.

$\mathrm{P}:$ Bah voilà.

E3 : Ah ouais.

P : Est-ce que votre façon de penser n'est pas influencée par la société ?

E1 : Bah non.

E2 : Si.

E4 : Bah si.

E1 : Bah non.

E3 : Bah si, les médias. 


\begin{tabular}{|c|}
\hline $\begin{array}{l}\text { P : La mode, les médias,... Vous ne faites pas forcément... Dans ce qu'on vous propose, vous } \\
\text { choisissez. Ça veut pas dire, peut-être, que tout vous est imposé, mais il y a quand même des } \\
\text { influences. On vous propose, la mode, les médias... Axel! }\end{array}$ \\
\hline E1: Bah la mode, on fait ce qu'on veut. \\
\hline E3 : Bah oui mais tu t'habilles en fonction de la mode. \\
\hline E1 : [inaudible] On s'en fiche de la mode. \\
\hline P : D'accord. Et la majorité fait quoi? \\
\hline E1 : Bah la mode mais... Il y a bien des gens... \\
\hline E2 : Oui, mais la majorité... \\
\hline $\begin{array}{l}\text { P : Donc vous êtes d'accord de dire que la société influence... Alors la mode vous convainc peut-être } \\
\text { pas, mais la société influence. Bon, donc là, ce que je vous ai donné c'est le Code Napoléon, c'est à } \\
\text { dire le code civil qui régit les lois, les règles de relation entre les personnes et de gestion des biens. } \\
\text { Et donc, on nous dit tout ce que la femme - je vous ai pas mis les articles hein, on vous a pas mis les } \\
\text { articles précis, mais on vous a résumé ce que la femme pouvait avoir comme droits, comme } \\
\text { obligations, comme possibilités. Et votre première réaction, ça a été quoi ? Vous m'avez dit quoi? }\end{array}$ \\
\hline E1 : Bah, c'est pas la faute de la société. \\
\hline $\mathrm{P}$ : Non, par rapport à ça. \\
\hline E1 : Ah ! Elles ont rien le droit de faire. \\
\hline $\begin{array}{l}\mathrm{P} \text { : Voilà, donc ce que je vous demande, justement, c'est de répondre à la question - du coup, bah les } \\
\text { femmes peuvent faire quoi ? Et du coup, ça nous donne une idée sur les possibilités de Mathilde. Et } \\
\text { pourquoi Mathilde, peut-être, réagit comme ça. }\end{array}$ \\
\hline
\end{tabular}

\section{NOTES}

1. Nous désignons au sens large par "geste de lecture " un mouvement réactif de l'imagination, de la sensibilité et de la pensée, la concrétisation d'une préoccupation du sujet-lecteur face au texte. Employée dans un sens métaphorique par l'écrivain C. Ollier (1975), dans un article éponyme, à propos du processus de lecture-écriture, l'expression suppose pour nous une pratique et une éducabilité, inscrite dans les codes d'une culture partagée, prenant sens dans et par le contexte scolaire. La notion de geste intègre la dimension corporelle de l'acte de lecture, ce qui rejoint la notion de « lisart » proposée par V. Larrivé (2014). Elle contient l'idée d'un mouvement qui s'adresse à un autre, lui donne existence et statut, tant il est vrai que la lecture a une dimension dialogique, ainsi que le rappelle T. Todorov $(1984$, p. 186) : « L'auteur est un "tu" et non pas un "il", un interlocuteur avec qui on débat de valeurs humaines". La notion enveloppe celle de geste appropriatif, dont B. Shawky-Milcent (2014) a proposé une description fine. Elle enveloppe aussi les activités fictionnalisantes du lecteur théorisées par M.-J. Fourtanier et G. Langlade (2006). La notion de geste ne se substitue pas à celle de compétence de lecture, elle se situe en amont. Elle contient en elle le devenir d'une compétence, tout en étant plus en prise avec le mouvement de la lecture, pour rendre compte de la combinaison, l'association, l'articulation des réactions, entre les différentes composantes de la lecture axiologique. Les gestes de lecture sont des réponses concrètes, situées, face à un écrit qui interpelle. Les champs gestuels dans lesquels on peut les regrouper se rapprochent des "postures de lecture", en tant que 
"schèmes pré-réflexifs disponibles" (Bucheton, 1999; 2006). Mais les "postures " désignent surtout un rapport de représentation au " comment faire ", elles procèdent d'une réflexion sur l'engagement dans la tâche, alors que les champs gestuels forment une topique de l'engagement personnel par rapport à une interpellation axiologique et esthétique du texte. Gestes et postures sont construits dans l'histoire personnelle et sociale du sujet (à fortiori l'histoire scolaire). Ils sont à la fois du côté du sujet dans un contexte donné, mais aussi de l'objet et de la situation. Tous deux évoluent au cours de la lecture. Pour une première réflexion sur la notion de geste de lecture, voir l'introduction à la séance 1 du séminaire Littéval: http://ouvroir-litt-arts.univgrenoble-alpes.fr/programmes/littvaleurs/seance-1-du-seminaire-liteval.

2. Voir aussi Dahhan (1996).

3. Un interdit est un «majeur dans l'état habituel d'imbécilité, de démence ou de fureur, même lorsque cet état présente des intervalles lucides " (art. 489) et qui est assimilé « au mineur, pour sa personne et pour ses biens » (art. 509).

4. Voir Collin (2015).

5. Financé par l'Idex pour la période 2017-2019, le projet interdisciplinaire Littéval regroupe 13 chercheurs de 7 laboratoires nationaux et internationaux. Il a pour objectif d'étudier les conditions de possibilité, les difficultés et les effets éducatifs d'un enseignement de la littérature par le questionnement éthique, de la fin du primaire au baccalauréat. Il s'agit tout d'abord de fonder épistémologiquement une approche des textes littéraires affranchie d'un substantialisme fort. Le second objectif est d'ébaucher un état des lieux socio-institutionnel des pratiques scolaires, en distinguant les influences liées aux valeurs de l'institution littéraire, celles liées aux habitus sociaux et celles liées aux valeurs contextuelles de la lecture en classe. Le troisième objectif est pédagogique et didactique. Il s'agit de construire des scénarios pédagogiques qui articulent entre elles plusieurs composantes de la lecture axiologique, selon des parcours diversifiés. La recherche vise l'émergence de compétences de lecture axiologiques chez les élèves et à établir une typologie des gestes professionnels de l'enseignant, dans la conduite du questionnement et l'accompagnement des discussions.

6. La séquence s'inscrit dans l'objet étude des programmes de 2015 pour le collège «Individu et société : confrontation de valeurs? ». D’une durée totale de 10 heures, elle comporte huit séances.

7. Il s'agit de la deuxième année du cycle 4 du collège, pour des élèves âgés de 13/14 ans.

8. L'Éspé (École supérieure du professorat et de l'éducation), qui assure la formation initiale des professeurs du premier et du second degré, est devenue en septembre 2019 l'Inspé (Institut national supérieur du professorat et de l'éducation).

9. Dispositif qui consiste à lire des textes avec les élèves, en révélant le contenu par étapes successives. Elaboré, dès 1982 par A. Béguin dans son ouvrage Lire-écrire: pratique nouvelle de la lecture au collège (L'école des loisirs), ce dispositif est ensuite développé et précisé par J.-L. Dufays, L. Gemenne et D. Ledur (2015 [1996]).

10. Les séances ont été enregistrées et les échanges ont été transcrits.

11. Dans les transcriptions qui suivent, les tours de parole sont numérotés. Chaque locuteur est désigné par ses initiales, le professeur par « $\mathrm{P}$ », les élèves par « $\mathrm{E}$ » avec un numéro qui les individualise (E1, E2, etc.). Les initiales sont suivies d'un point et sont 
reprises à chaque tour de parole. Nous avons opté pour une transcription orthographique standard sans avoir renormalisé la parole. À des fins de simplification et de lisibilité, ni les pauses ni les liaisons ne sont notées.

12. L'opposition de cet élève exprime des valeurs individualistes qu'on ne peut pas rapporter avec certitude à son milieu socio-culturel. En tout cas ce dernier n'est pas spécifiquement marqué socialement ou économiquement. À cette difficulté se sont ajoutés pour ce groupe plusieurs obstacles lexicaux à la compréhension du document, ce qui a retardé la mise en relation avec le texte de Maupassant.

13. Il s'agit d'un questionnaire d'enquête mis en ligne entre le 8 février et le 30 juin 2019, qui s'inscrit dans le cadre de la recherche "Littérature et valeurs ». L'objet de l'enquête était caché aux sondés derrière un intitulé général : «L'enseignement des textes littéraires au collège et au lycée ». Il s'agissait de cerner quelle est la place du questionnement axiologique et quelles sont ses modalités, en comparant les pratiques déclarées avec une réflexion didactique en acte sur un support textuel donné. Sur 802 répondants, 387 sont allés au bout de l'exercice. Cinq d'entre eux évoquent le recueil des impressions de lecture des élèves, en guise d'objectifs d'apprentissage ou de problématique.

\section{RÉSUMÉS}

L'article interroge la conception de la lecture littéraire comme «enroulement spiralaire » (Shawky-Milcent, 2014), au regard d'un dispositif à priori éloigné de l'approche du sujet lecteur, en raison de son objet et du guidage qu'il implique. Il s'agit de susciter une relecture de la nouvelle de Maupassant (1885) «La Parure » en classe de quatrième, au regard d'un intertexte juridique. Après avoir posé le cadre théorique de deux composantes de la lecture axiologique, l'article présente le dispositif et analyse les moments de classe concernés, du point de vue des gestes de lecture et des gestes professionnels. Il discute ensuite la prise en compte d'un tel dispositif au titre de l'enroulement spiralaire, afin de questionner en retour la didactique du sujet lecteur sur les savoirs contextuels, la démarche d'enseignement et le guidage.

The article questions the conception of literary reading as a "spiral coil" (Shawky-Milcent, 2014), in relation with a device that is a priori far removed from the approach of the reading subject, because of its object and the guidance it implies. In the Year $9 / 8^{\text {th }}$ grade, the aim, the aim is to encourage a re-reading of Maupassant's short story "La Parure", with regard to a legal intertext. After establishing the theoretical framework of two components of axiological reading, the article presents the device and analyses the class moments concerned, from the point of view of reading and professional gestures. Then, it discusses the consideration of such a device under spiral winding, in order to question in return the didactics of the reader subject on contextual knowledge, the teaching approach and guidance. 
INDEX

Keywords : reading, literature, education, civic values, law, history

Mots-clés : Lecture littéraire, éducation, contextualisation, valeurs, droit, histoire

\section{AUTEUR}

\section{NICOLAS ROUVIÈRE}

Université Grenoble Alpes, Litt\&Arts CNRS, F-38400 Saint-Martin-d'Hères, France 